antituberculosis therapy and fever resolved after 45 days. A nurse is going to her house daily to check the patient's adherence during the one year treatment.

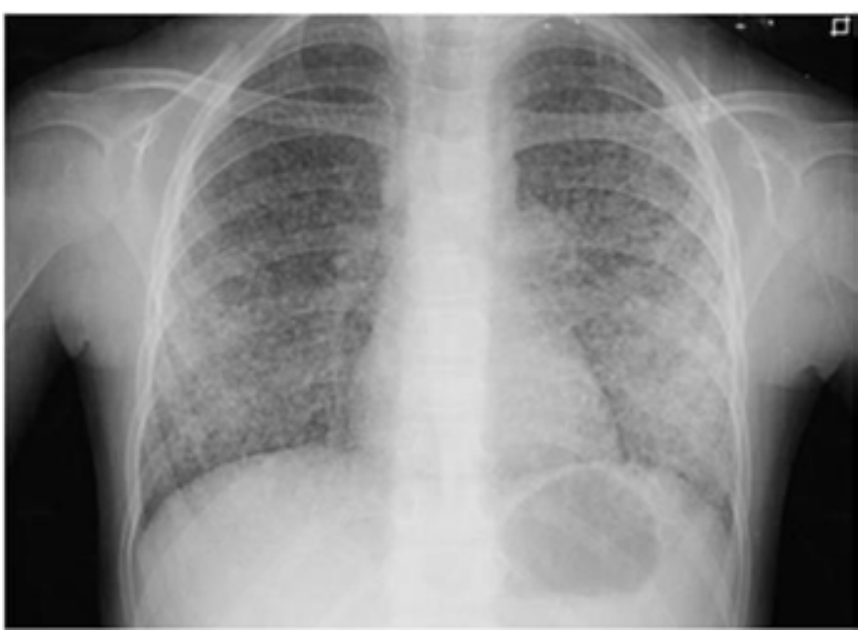

Abstract 953 Figure 1 Chest radiography of miliary tuberculosis

Conclusion The diagnosis of MT can be clearly invoked with a simple and inexpensive investigation, even in an immunocompetent child. The typical image in the chest radiography is the most important reason to report this case.

\section{PLEURAL EFFUSION AND ASCITES: AN UNUSUAL COMPLICATION OF HEPATITIS A}

doi:10.1136/archdischild-2012-302724.0954

TI El-Azzabi, V Malpani. Paediatrics, Child Health Institute, Al Ain Hospital, Al Ain, United Arab Emirates

Background and Aims To the best of our knowledge, we are reporting the first case from United Arab Emirates in the literature of Hepatitis A associated with pleural effusion and ascites. Hepatitis $A$ is the most common cause of viral hepatitis in childhood and a major health problem in the developing world. Pleural effusion and ascites are very rare extrahepatic complications of hepatitis $\mathrm{A}$. There have been only a few case reports in the literature of the two complications. The etiology is not clearly understood, but they tend to undergo spontaneous resolution and do not warrant specific diagnostic or therapeutic measures.

Methods Information collected from the hospital electronic case notes (Cerner).

Results Our case is a three years old Afghani boy who presented with fever and jaundice for two days. There was no history of recent travel abroad. Investigations revealed a high DIRECT bilirubin level and elevated liver enzymes. Hepatitis A virus immunoglobulin M (HAV IgM) was detected. During the course of hepatitis A, the child developed clinically significant and symptomatic bilateral pleural effusion associated with ascites. The diagnosis was confirmed with chest x-ray and ultrasonography. Lowest Albumin level was $23 \mathrm{~g} / \mathrm{l}$. Both pleural effusion and ascites resolved spontaneously without intervention.

Conclusions In patients with jaundice and pleural effusion and/or ascites, Hepatitis $\mathrm{A}$ is an important differential diagnosis. Both conditions are self-limited. Pleural and/or peritoneal diagnostic tapping is not warranted. Research is required to explore the underlying pathogenesis of the association.
É Karaszi, K Kalocsai, K Kardics, Z Liptai, A Trethon. Pediatric Infectology, St. László Hospital, Budapest, Hungary

Background and Aims A ten year-old girl with hyper IgE syndrome caused by DOCK8 mutation was admitted to our hospital due to neuropsychiatric symptoms. Cranial MRI revealed multifocal cerebral lesions. Our aim was to clarify the etiology of these lesions by extended microbiology tests and comprehensive search in the literature then provide her with proper treatment options.

Methods Multiple blood and cerebrospinal fluid samples and were examined for bacterial and fungal culture, Aspergillus and Cryptococcus antigen, HSV, CMV, Mycobacterium and Toxoplasma PCR, panfungal PCR and for Toxocara and E. hystolytica serology. Brain biopsy was also done for histology, bacterial and fungal culture.

Results All diagnostic assays showed negative results therefore causative agents could not be identified For treatment, ceftriaxon and metronidazole combination was initially used accompanied by slight clinical and neuroradiological progression. Considering the possible presence of vascular brain lesions, high dose parenteral steroid treatment was introduced together with preemptive parenteral voriconazol therapy. Further progression in the clinical and radiological status was observed. Although there is no report of cerebral toxoplasmosis in this disorder, empirical antitoxoplasma treatment was initiated with significant clinical improvement and radiological regression after 6-week therapy. Retrospective tests of CSF for Toxoplasma serology showed IgG titer increment.

Conclusion To our knowledge this is the first paper on cerebral toxoplasmosis in hyper IgE syndrome to date. In case of cerebral lesions in these patients Toxoplasma reactivation should be considered inspite of negative Toxoplasma PCR and antitoxoplasma treatment sould be introduced in the absence of other etiologic factor.

\section{INFANT BOTULISM DUE TO HONEY INGESTATION}

doi:10.1136/archdischild-2012-302724.0956

${ }^{1} \mathrm{~F}$ Abbasi, ${ }^{2} \mathrm{P}$ Vahdani, ${ }^{2} \mathrm{MR}$ Adhami Mojarad, ${ }^{3} \mathrm{~S}$ Korooni. ${ }^{1}$ Bushehr University of Medical Sciences, Bushehr; ${ }^{2}$ Infectious Diseases and Tropical Medicine Research Center, Shaheed Beheshti Medical University, Tehran; ${ }^{3}$ Shiraz University of Medical Sciences, Shiraz, Iran

Background and Aims Botulism is a neuroparalytic disease caused by neurotoxins produced by the bacteria Clostridium botulinum. This neurotoxin inhibits the normal release of acetylcholine in the synaptic cleft, inducing presynaptic neuromuscular blockade. Infant botulism results from the absorption of heat-labile neurotoxin produced in situ by ingested Clostridium botulinum. Honey and environmental exposure are the main sources of acquisition of the organism.

Patient The patient was a 6-month-old girl with bilateral ptosis, muscle weakness, constipation and history of honey consumption. Lumbar puncture (LP), electromyography (EMG), nerve conduction study (NVS) and brain magnetic resonance imaging (MRI) were performed that all were normal. Stool evaluation for botulinum toxin was positive for toxin-A. Due to delayed diagnosis and improvement of general condition with conservative management botulinum anti-toxins did not started for her. After several days symptoms graduallay improved and she was discharged with good general condition.

Conclusion Botulinum should be considered in every patient with weakness and ptosis. Botulism evaluation and approporiate management should be done.

\section{MULTIPLE HUGE HYDATID CYSTS OF LIVER}

